\title{
PROLONGATION OF SYMMETRIC KILLING TENSORS AND COMMUTING SYMMETRIES OF THE LAPLACE OPERATOR
}

\author{
J.-P. MICHEL, P. SOMBERG AND J. ŠILHAN
}

\begin{abstract}
We determine the space of commuting symmetries of the Laplace operator on pseudo-Riemannian manifolds of constant curvature, and derive its algebra structure. Our construction is based on the Riemannian tractor calculus, allowing to construct a prolongation of the differential system for symmetric Killing tensors. We also discuss some aspects of its relation to projective differential geometry.
\end{abstract}

Key words: Killing tensors, Prolongation of PDEs, Commuting symmetries of Laplace operator.

MSC classification: 35R01, 53A20, 58J70, 35J05 .

\section{INTRODUCTION}

The Laplace operator is one of the cornerstones of geometrical analysis on pseudo-Riemannian manifolds, and there exists a close relationship between spectral properties of the Laplace operator and local as well as global invariants of the underlying pseudo-Riemannian manifold.

The question of conformal symmetries of the Yamabe-Laplace operator $\Delta$ on conformally flat spaces has been solved in [8]. A differential operator $D$ is a conformal symmetry of $\Delta$ provided $[\Delta, D] \in(\Delta)$, where $(\Delta)$ is the left ideal generated by $\Delta$ in the algebra of differential operators. These operators $D$ are called conformal symmetries, because they preserve the kernel of $\Delta$. On a given flat conformal manifold $M$, there is a bijection between the vector space of symmetric conformal Killing tensors and the quotient of the space of conformal symmetries by $(\Delta)$. Notice that the space of symmetric conformal Killing tensors is the solution space of a conformally invariant system of overdetermined partial differential equations, which turns out to be locally finite dimensional.

In the present paper, we classify the commuting symmetries of the Laplace operator on pseudo-Riemannian manifolds of constant curvature, i.e., manifolds locally isometric to a space form. In this case the Laplace operator differs from the Yamabe-Laplace operator by a multiple of the identity operator. With an abuse of notation, we denote both by $\Delta$. The eigenspaces of the Laplace operator are preserved by commuting symmetries, i.e., by linear differential operators $D$ commuting 
with the Laplace operator:

$$
[\Delta, D]=0
$$

The vector space of commuting symmetries is generated by Killing vector fields and their far reaching generalization called symmetric Killing tensors, or Killing tensors for short. Their composition as differential operators provides an algebra structure that we determine.

Killing 2-tensors on pseudo-Riemannian manifolds are the most studied among Killing tensors, and they play a key role in the separation of variables of the Laplace equation. The construction of commuting symmetries out of Killing 2-tensors is well-known in number of geometrical situations [7], in particular on constant curvature manifolds. Higher Killing tensors give integrals of motion for the geodesic equation and contribute to its integrability. They can be regarded as hidden symmetries of the underlying pseudo-Riemannian manifold. Killing tensors themselves are solutions of an invariant system of PDEs, and trace-free Killing tensors are special examples of conformal Killing tensors.

As a technical tool we introduce, and to a certain extent develop, the Riemannian tractor calculus, focusing mainly on manifolds of constant curvature. This allows a uniform description of the prolongation of the invariant system of PDEs for Killing tensors, and plays a key role in our analysis of the correspondence between commuting symmetries of the Laplace operator and Killing tensors. In particular, we obtain an explicit version of the identification in [15] (see also [17]) of the space of fixed valence Killing tensors with a representation of the general linear group.

The Riemannian tractor calculus can be interpreted as the tractor calculus for projective parabolic geometry in a scale corresponding to a metric connection in the projective class of affine connections. Restricting to locally flat special affine connections, Einstein metric connections in the projective class correspond to manifolds of constant curvature [11. Since the Killing equations on symmetric tensor fields are projectively invariant [9], we can use the invariant tractor calculus in projective parabolic geometry to construct commuting symmetries. Note the projective invariance explains that the space of Killing tensors carries a representation of the general linear group.

As for the style of presentation and exposition, we have tried to make the paper accessible to a broad audience, with basic knowledge in Riemannian geometry. Following this perspective, the structure of our paper goes as follows. After setting the conventions in Section 2, we introduce in Section 3 the rudiments of Riemannian tractor calculus. The core of the article is in Section 4, where we construct the prolongation of the differential system for Killing tensors and derive the space of differential operators preserving the spectrum of the Laplace operator. Afterwards, we determine the underlying structure of associative 
algebra on this space, induced by the composition of differential operators. We compute explicit formulas for commuting symmetries of order at most 3. In special cases we compare commuting symmetries with conformal symmetries constructed in [8]. In Section 5, we interpret our results in terms of the holonomy reduction of a Cartan connection in projective parabolic geometry and its restriction on a curved orbit equipped with Einstein metric.

The authors are grateful to A. Čap, M. G. Eastwood and A. R. Gover for their suggestion on the interpretation of our results in the framework of projective parabolic geometry.

\section{Notation AND CONVENTIONS}

Let $(M, g)$ be a smooth pseudo-Riemannian manifold. Throughout the paper we employ the Penrose's abstract index notation and write $\mathcal{E}^{a}$ to denote the space of smooth sections of the tangent bundle $T M$ on $M$, and $\mathcal{E}_{a}$ for the space of smooth sections of the cotangent bundle $T^{*} M$. We also write $\mathcal{E}$ for the space of smooth functions. All tensors considered are assumed to be smooth. With abuse of notation, we will often use the same symbols for the bundles and their spaces of sections. The metric $g_{a b}$ will be used to identify $T M$ with $T^{*} M$. We shall assume that the manifold $M$ has dimension $n \geq 2$.

An index which appears twice, once raised and once lowered, indicates the contraction. The square brackets $[\cdots]$ will denote skewsymmetrization of enclosed indices, while the round brackets $(\cdots)$ will indicate symmetrization.

We write $\nabla$ for the Levi-Civita connection corresponding to $g_{a b}$. Then the Laplacian $\Delta$ is given by $\Delta=g^{a b} \nabla_{a} \nabla_{b}=\nabla^{b} \nabla_{b}$. Since the Levi-Civita connection is torsion-free, the Riemannian curvature $R_{a b}{ }^{c} d$ is given by $\left[\nabla_{a}, \nabla_{b}\right] v^{c}=R_{a b}^{c} v^{d}$, where $[\cdot, \cdot]$ indicates the commutator bracket. The Riemannian curvature can be decomposed in terms of the totally trace-free Weyl curvature $C_{a b c d}$ and the symmetric Schouten tensor $\mathrm{P}_{a b}$,

$$
R_{a b c d}=C_{a b c d}+2 g_{c[a} \mathrm{P}_{b] d}+2 g_{d[b} \mathrm{P}_{a] c} .
$$

We will refer to $\mathrm{P}_{a b}$ as to Riemannian Schouten tensor to distinguish from the projective Schouten tensor introduced later. We define $\mathrm{J}:=$ $\mathrm{P}_{a}^{a}$, so that $\mathrm{J}=\frac{\mathrm{Sc}}{2(n-1)}$ with Sc the scalar curvature.

Throughout the paper we work (if not stated otherwise) on manifolds of constant curvature, i.e., locally symmetric spaces with parallel curvature $R_{a b c d}=\frac{4}{n} \mathrm{~J} g_{c[a} g_{b] d}$, cf. [18]. This means that $\mathrm{J}$ is parallel for the Levi-Civita connection $\nabla$. In signature $(p, q), M$ is then locally isomorphic to $G / H$, where $G=S O(p+1, q)$ and $H=S O(p, q)$ if $J>0$, $G=S O(p, q+1)$ and $H=S O(p-1, q+1)$ if $J<0, G=E(p, q)$ and $H=S O(p, q)$ if $J=0$. Here we denote by $E(p, q)$ the group of pseudo-Euclidean motions on $\mathbb{R}^{p, q}$. 


\section{Tractor Calculus in Riemannian GeOmetry}

The notion of associated tractor bundles is well-known in the category of parabolic geometries. We refer to [5] for a review with many applications. In the present section we introduce and develop rudiments of a class of tractor bundles in the category of pseudo-Riemannian manifolds, in a close analogy with tractor calculi in parabolic geometries.

Recall we assume $M$ has constant curvature, i.e. $R_{a b c d}=\frac{4}{n} \mathrm{~J} g_{c[a} g_{b] d}$ with J constant. We define the Riemannian standard tractor bundle (standard tractor bundle, for short) as $\mathcal{T}:=\mathcal{L} \oplus T M$ where $\mathcal{L}$ denotes the trivial bundle over $M$.

The Levi-Civita connection $\nabla_{a}$ induces a connection on $\mathcal{T}$, which is trivial on $\mathcal{L}$. The tractor connection is another connection on $\mathcal{T}$, also denoted (with an abuse of notation) by $\nabla_{a}$, and defined by

$$
\nabla_{a}\left(\begin{array}{c}
f \\
\mu^{b}
\end{array}\right)=\left(\begin{array}{c}
\nabla_{a} f-\mu_{a} \\
\nabla_{a} \mu^{b}+\frac{2}{n} \mathrm{~J} f \delta_{a}^{b}
\end{array}\right)
$$

where $f \in \mathcal{E}, \mu^{b} \in \mathcal{E}^{b}$. In the first line, we use the isomorphism $T M \cong T^{*} M$. The dual connection on the dual bundle $\mathcal{T}^{*}:=T^{*} M \oplus \mathcal{L}$, denoted also by $\nabla_{a}$, is given by

$$
\nabla_{a}\left(\begin{array}{c}
\nu_{b} \\
f
\end{array}\right)=\left(\begin{array}{c}
\nabla_{a} \nu_{b}+f g_{a b} \\
\nabla_{a} f-\frac{2}{n} \mathrm{~J} \nu_{a}
\end{array}\right)
$$

where $\nu_{b} \in \mathcal{E}_{b}$ and $f \in \mathcal{E}$. A direct computation shows that the curvature of the tractor connection $\nabla$ is trivial, i.e., the tractor connection $\nabla$ is flat. Note this connection differs from the tractor connection induced by the Cartan connection, as discussed in [5, section 1.5].

The bundle $\mathcal{T}$ is equipped with the symmetric bilinear form $\langle$,$\rangle ,$

$$
\left\langle\left(\begin{array}{c}
f \\
\mu^{b}
\end{array}\right),\left(\begin{array}{c}
\bar{f} \\
\bar{\mu}^{b}
\end{array}\right)\right\rangle=\frac{2}{n} \mathrm{~J} f \bar{f}+\mu^{b} \bar{\mu}_{b}
$$

which is invariant with respect to the tractor connection $\nabla$. For $\mathrm{J} \neq 0$, this form is non-degenerate and called the tractor metric. It yields then an isomorphism $\mathcal{T} \cong \mathcal{T}^{*}$.

We define the adjoint tractor bundle as $\mathcal{A}:=\wedge^{2} \mathcal{T}=T M \oplus \wedge^{2} T M$ and we extend the tractor connection from $\mathcal{T}$ to $\mathcal{A}$ by the Leibniz rule. Similarly, we obtain an induced tractor connection on $\mathcal{A}^{*}=$ $\wedge^{2} T^{*} M \oplus T^{*} M$. Explicitly, these connections are given by the formulas

$\nabla_{a}\left(\begin{array}{c}\varphi^{b} \\ \psi^{b c}\end{array}\right)=\left(\begin{array}{c}\nabla_{a} \varphi^{b}-2 \psi_{a}^{b} \\ \nabla_{a} \psi^{b c}+\frac{2}{n} \mathrm{~J} \delta_{a}^{b} \varphi^{c]}\end{array}\right) \quad$ and $\quad \nabla_{a}\left(\begin{array}{c}\mu_{b c} \\ \omega_{b}\end{array}\right)=\left(\begin{array}{c}\nabla_{a} \mu_{b c}+2 g_{a[b} \omega_{c]} \\ \nabla_{a} \omega_{b}-\frac{4}{n} \mathrm{~J} \mu_{a b}\end{array}\right)$

where $\left(\varphi^{b}, \psi^{b c}\right) \in \Gamma(\mathcal{A})$, i.e. $\varphi^{b} \in \mathcal{E}^{b}$ and $\psi^{b c} \in \mathcal{E}^{[b c]}$, and $\left(\mu_{b c}, \omega_{b}\right) \in$ $\Gamma\left(\mathcal{A}^{*}\right)$, i.e. $\mu_{b c} \in \mathcal{E}_{[b c]}$ and $\omega_{b} \in \mathcal{E}_{b}$. 
We extend the tractor connection to the tensor product bundle $(\otimes \mathcal{T}) \otimes$ $\left(\otimes \mathcal{T}^{*}\right)$ by the Leibniz rule. The resulting connection is again flat, denoted by $\nabla$ and termed the tractor connection. Further, the tractor and Levi-Civita connections induce the connection

$$
\nabla_{a}: \mathcal{E}_{b \ldots d} \otimes \Gamma(W) \rightarrow \mathcal{E}_{a b \ldots d} \otimes \Gamma(W)
$$

for any tractor subbundle $W \subseteq(\otimes \mathcal{T}) \otimes\left(\otimes \mathcal{T}^{*}\right)$, i.e., any subbundle preserved by the tractor connection. This coupled Levi-Civita-tractor connection allows to extend all natural operators, e.g. the Laplace operator $\Delta$, to tensor-tractor bundles.

The invariant pairing on $\mathcal{A}$ induced by (4) is given by the formula

$$
\left\langle\left(\begin{array}{c}
\varphi^{b} \\
\psi^{b c}
\end{array}\right),\left(\begin{array}{c}
\bar{\varphi}^{b} \\
\bar{\psi}^{b c}
\end{array}\right)\right\rangle=\frac{1}{n} \mathrm{~J} \varphi^{a} \bar{\varphi}_{a}+\psi^{a b} \bar{\psi}_{a b} .
$$

For $\mathrm{J} \neq 0$, this defines a metric on $\mathcal{A}$ and $\mathcal{A} \cong \mathcal{A}^{*}$. Moreover, there is a Lie algebra structure $[\cdot, \cdot]: \mathcal{A} \otimes \mathcal{A} \rightarrow \mathcal{A}$ given by

$$
\left[\left(\begin{array}{c}
\varphi^{b} \\
\psi^{b c}
\end{array}\right),\left(\begin{array}{c}
\bar{\varphi}^{b} \\
\bar{\psi}^{b c}
\end{array}\right)\right]=\left(\begin{array}{c}
\varphi_{r} \bar{\psi}^{r a}-\bar{\varphi}_{r} \psi^{r a} \\
-2 \psi^{r b} \bar{\psi}_{r}^{c]}-\frac{1}{n} \mathrm{~J} \varphi^{[b} \bar{\varphi}^{c]}
\end{array}\right)
$$

which is also invariant under the tractor connection.

Remark 3.1. Tractor connections can be defined on any Riemannian manifold. For example for $\varphi^{b} \in \mathcal{E}^{b}$ and $\psi^{b c} \in \mathcal{E}^{[b c]}$, we can define $\nabla$ on $\mathcal{A}$ by

$$
\nabla_{a}\left(\begin{array}{c}
\varphi^{b} \\
\psi^{b c}
\end{array}\right)=\left(\begin{array}{c}
\nabla_{a} \varphi^{b}-2 \psi_{a}^{b} \\
\nabla_{a} \psi^{b c}+\frac{1}{2} R_{a s}^{b c} \varphi^{s}
\end{array}\right)
$$

This definition originates in the work by B. Kostant [14. We observe that, for a Killing vector field $k^{a} \in \mathcal{E}^{a}$, its prolongation

$$
K=\left(\begin{array}{c}
k^{a} \\
\frac{1}{2} \nabla^{[a} k^{b]}
\end{array}\right) \in \Gamma(\mathcal{A})
$$

is parallel for the tractor connection. Hence, any isometry is locally determined by its first jet.

We shall use the abstract index notation for the adjoint tractor bundle as follows: $\Gamma(\mathcal{T})$ will be denoted by $\mathcal{E}^{A}$ and $\Gamma(\mathcal{A})$ will be denoted by $\mathcal{E}^{\mathbf{A}}$ where $\mathbf{A}=\left[A^{1} A^{2}\right]$. Similarly $\mathcal{E}_{A}=\Gamma\left(\mathcal{A}^{*}\right)$ and $\mathcal{E}_{\mathbf{A}}=\Gamma\left(\mathcal{A}^{*}\right)$. That is, we use bold face capital indices for adjoint tractor bundle and its dual.

There is a convenient way to treat bundles $\mathcal{T}$ and $\mathcal{T}^{*}$, based on the so-called injectors, or tensor-tractor frame, denoted by $Y^{A}, Z_{a}^{A}$ for $\mathcal{T}$ and denoted by $Y_{A}, Z_{A}^{a}$ for $\mathcal{T}^{*}$. These are defined by

$$
\left(\begin{array}{c}
f \\
\mu^{b}
\end{array}\right)=Y^{A} f+Z_{b}^{A} \mu^{b}, \quad\left(\begin{array}{c}
\nu_{b} \\
f
\end{array}\right)=Z_{A}^{b} \nu_{b}+Y_{A} f
$$


and their contractions are $Y^{A} Y_{A}=1, Z_{a}^{A} Z_{A}^{b}=\delta_{a}^{b}$ and $Y^{A} Z_{A}^{b}=Z_{a}^{A} Y_{A}=$ 0 . The covariant derivatives in (2) and (3) are then encoded in covariant derivatives of these injectors,

$$
\begin{array}{ll}
\nabla_{c} Y^{A}=\frac{2}{n} \mathrm{~J} Z_{a}^{A} \delta_{c}^{a}, & \nabla_{c} Z_{a}^{A}=-Y^{A} g_{a c} \\
\nabla_{c} Z_{A}^{a}=-\frac{2}{n} \mathrm{~J} Y_{A} \delta_{c}^{a}, & \nabla_{c} Y_{A}=Z_{A}^{a} g_{c a} .
\end{array}
$$

We denote the tractor pairing (41) by $h_{A B} \in \mathcal{E}_{(A B)}$ which has the explicit form

$$
h_{A B}=\frac{2}{n} \mathrm{~J} Y_{A} Y_{B}+Z_{A}^{a} Z_{B}^{b} g_{a b} .
$$

Injectors for the adjoint tractor bundle $\mathcal{E}^{\mathbf{A}}$ are $\mathbb{Y}_{a}^{\mathbf{A}}=Y^{\left[A^{1}\right.} Z_{a}^{\left.A^{2}\right]}$ and $\mathbb{Z}_{\mathbf{a}}^{\mathbf{A}}=Z_{a^{1}}^{\left[A^{1}\right.} Z_{a^{2}}^{\left.A^{2}\right]}$, injectors for the dual bundle $\mathcal{E}_{\mathbf{A}}$ are $\mathbb{Y}_{\mathbf{A}}^{a}=Y_{\left[A^{1}\right.} Z_{\left.A^{2}\right]}^{a}$ and $\mathbb{Z}_{\mathbf{A}}^{\mathbf{a}}=Z_{\left[A^{1}\right.}^{a^{1}} Z_{\left.A^{2}\right]}^{a^{2}}$. That is,

$$
\left(\begin{array}{c}
\varphi^{b} \\
\psi^{\mathbf{a}}
\end{array}\right)=\varphi^{b} \mathbb{Y}_{b}^{\mathbf{A}}+\psi^{\mathbf{a}} \mathbb{Z}_{\mathbf{a}}^{\mathbf{A}} \quad\left(\begin{array}{c}
\mu_{\mathbf{a}} \\
\omega_{b}
\end{array}\right)=\mu_{\mathbf{a}} \mathbb{Z}_{\mathbf{A}}^{\mathbf{a}}+\omega_{b} \mathbb{Y}_{\mathbf{A}}^{b}
$$

where $\mathbf{a}=\left[a^{1} a^{2}\right]$. The only nonzero contractions are $\mathbb{Y}_{a}^{\mathbf{A}} \mathbb{Y}_{\mathbf{A}}^{b}=\frac{1}{2} \delta_{a}^{b}$ and $\mathbb{Z}_{\mathbf{a}}^{\mathbf{A}} \mathbb{Z}_{\mathbf{A}}^{\mathbf{c}}=\delta_{\left[a^{1}\right.}^{c^{1}} \delta_{\left.a^{2}\right]}^{c^{2}}$. The covariant derivatives (5) are then equivalent to

$$
\begin{array}{ll}
\nabla_{c} \mathbb{Y}_{b}^{\mathbf{A}}=\frac{2 \mathrm{~J}}{n} \mathbb{Z}_{a b}^{\mathbf{A}} \delta_{c}^{a}, & \nabla_{c} \mathbb{Z}_{\mathbf{a}}^{\mathbf{A}}=-2 \mathbb{Y}_{\left[a^{2}\right.}^{\mathbf{A}} g_{\left.a^{1}\right] c}, \\
\nabla_{c} \mathbb{Z}_{\mathbf{A}}^{\mathbf{a}}=-\frac{4 \mathrm{~J}}{n} \mathbb{Y}_{\mathbf{A}}^{\left[a^{2} \delta_{c}^{\left.a^{1}\right]},\right.} & \nabla_{c} \mathbb{Y}_{\mathbf{A}}^{b}=\mathbb{Z}_{\mathbf{A}}^{a b} g_{c a} .
\end{array}
$$

and the pairing (77) on $\mathcal{E}^{\mathbf{A}}$ can be written as

$$
h_{\mathbf{A B}}=\frac{4}{n} \mathrm{~J} \mathbb{Y}_{\mathbf{A}}^{a} \mathbb{Y}_{\mathbf{B}}^{b} g_{a b}+\mathbb{Z}_{\mathbf{A}}^{\mathbf{a}} \mathbb{Z}_{\mathbf{B}}^{\mathbf{b}} g_{a^{1} b^{1}} g_{a^{2} b^{2}} .
$$

A crucial ingredient in our construction will be the differential operator

$$
\mathbb{D}_{\mathbf{A}}: \mathcal{E}_{b_{1} \ldots b_{s}} \otimes \Gamma(W) \rightarrow \mathcal{E}_{b_{1} \ldots b_{s}} \otimes \Gamma\left(\mathcal{A}^{*} \otimes W\right)
$$

for a tractor subbundle $W \subseteq(\otimes \mathcal{T}) \otimes\left(\otimes \mathcal{T}^{*}\right)$. This operator is closely related to the so-called fundamental derivative [5]. It is defined as follows: for $f \in \Gamma(W)$, we put

$$
\mathbb{D}_{\mathbf{A}} f=\left(\begin{array}{c}
0 \\
2 \nabla_{a} f
\end{array}\right) \in \Gamma\left(\mathcal{A}^{*} \otimes W\right),
$$

and for $\varphi_{b} \in \mathcal{E}_{b}$, we put

$$
\mathbb{D}_{\mathbf{A}} \varphi_{b}=\left(\begin{array}{c}
2 g_{b\left[a^{1}\right.} \varphi_{\left.a^{2}\right]} \\
2 \nabla_{a} \varphi_{b}
\end{array}\right) \in \mathcal{E}_{b} \otimes \Gamma\left(\mathcal{A}^{*}\right) .
$$

Then we extend $\mathbb{D}_{\mathbf{A}}$ to all tensor-tractor bundles by the Leibniz rule. Using injectors (13), the formulas (17) and (18) are given by

$$
\begin{aligned}
& \mathbb{D}_{\mathbf{A}} f=2 \mathbb{Y}_{\mathbf{A}}^{a} \nabla_{a} f, \\
& \mathbb{D}_{\mathbf{A}} \varphi_{b}=2 \mathbb{Y}_{\mathbf{A}}^{a} \nabla_{a} \varphi_{b}+\mathbb{Z}_{\mathbf{A}}^{\mathbf{a}} 2 g_{b\left[a^{0}\right.} \varphi_{\left.a^{1}\right]}
\end{aligned}
$$


where $\mathbf{a}=\left[a^{1} a^{2}\right]$.

Theorem 3.2. Let $M$ be a manifold of constant curvature. The operator $\mathbb{D}_{\mathbf{A}}$ commutes with the coupled Levi-Civita-tractor connection $\nabla_{c}$,

$$
\nabla_{c} \mathbb{D}_{\mathbf{A}}=\mathbb{D}_{\mathbf{A}} \nabla_{c}: \mathcal{E}_{b_{1} \ldots b_{s}} \otimes \Gamma(W) \rightarrow \mathcal{E}_{c \mathbf{A} b_{1} \ldots b_{s}} \otimes \Gamma(W) .
$$

Proof. Since the tractor connection is flat, it is sufficient to prove the statement for $W$ equal to the trivial line bundle. We present two versions of the proof.

First, one can easily show by direct computation (using (14) and (19)) that the explicit formulas for the compositions $\nabla_{c} \mathbb{D}_{\mathbf{A}}$ and $\mathbb{D}_{\mathbf{A}} \nabla_{c}$ are the same when acting on $f \in \mathcal{E}$ and $\varphi_{b} \in \mathcal{E}_{b}$. Hence the formulas agree on any tensor bundle.

Alternatively, recall that for a Killing vector field $k^{a} \in \mathcal{E}^{a}$, its prolongation $K^{\mathbf{A}} \in \Gamma(\mathcal{A})$ is parallel, see (9). We further observe that $L_{k}=K^{\mathbf{A}} \mathbb{D}_{\mathbf{A}}$ is the Lie derivative along $k^{a}$ when acting on tensor bundles. Since $L_{k}$ commutes with the covariant derivative and the space of Killing vector fields on manifolds with constant curvature has dimension equal to $\operatorname{dim}(\mathcal{A})=n+\frac{1}{2} n(n-1)$, the statement follows.

As a consequence of the previous theorem, $\mathbb{D}_{\mathbf{A}}$ commutes also with the Laplace operator $\Delta$ on functions, forms, etc. Let us make this result more general and more precise. Assume $F: \Gamma\left(U_{1}\right) \rightarrow \Gamma\left(U_{2}\right)$ is a Riemannian invariant linear differential operator, acting between tensor bundles $U_{1}, U_{2}$. Then, it can be written in terms of the metric, the Levi-Civita connection $\nabla$ and the curvature J. Regarding $\nabla$ in the formula for $F$ as the coupled Levi-Civita-tractor connection, we obtain the operator $F^{\nabla}: \Gamma\left(\mathcal{A}^{*} \otimes U_{1}\right) \rightarrow \Gamma\left(\mathcal{A}^{*} \otimes U_{2}\right)$. Using Theorem 3.2 and $\nabla_{a} \mathrm{~J}=\nabla_{a} g=0$, we get the following

Corollary 3.3. Let $F: \Gamma\left(U_{1}\right) \rightarrow \Gamma\left(U_{2}\right)$ be a Riemannian invariant linear differential operator on the manifold $M$. Then $\mathbb{D}_{\mathbf{A}}$ commutes with F, i.e.,

$$
\mathbb{D}_{\mathbf{A}} \circ F=F^{\nabla} \circ \mathbb{D}_{\mathbf{A}}: \Gamma\left(U_{1}\right) \rightarrow \Gamma\left(\mathcal{A}^{*} \otimes U_{2}\right) .
$$

\section{Commuting Symmetries of the Laplace operator}

Definition 4.1. Let $U$ be a tensor bundle and $F: \Gamma(U) \rightarrow \Gamma(U)$ be a linear differential operator on $M$. A commuting symmetry of the operator $F$ is a linear differential operator $\mathcal{D}$ fulfilling $\mathcal{D} F=F \mathcal{D}$.

We are interested in commuting symmetries of the Laplace operator $F=\Delta$ on functions. They form a subalgebra of the associative algebra of linear differential operators acting on $\mathcal{E}$. The commuting symmetries are particular case of the conformal symmetries of $\Delta$, studied in [8]. The exposition in the rest of the section closely follows the one given in 8 . 
Let $\ell$ be a non-negative integer. A linear $\ell$-th order differential operator acting on functions can be written as

$$
\mathcal{D}=V^{a_{1} \ldots a_{\ell}} \nabla_{a_{1}} \ldots \nabla_{a_{\ell}}+\operatorname{LOTS},
$$

where "LOTS" stands for lower order terms in $\mathcal{D}$ and its principal symbol $V^{a_{1} \ldots a_{\ell}}$ is symmetric in its indices, $V^{a_{1} \ldots a_{\ell}}=V^{\left(a_{1} \ldots a_{\ell}\right)}$.

Definition 4.2. A Killing tensor on $M$ is a symmetric tensor field $V^{a_{1} \ldots a_{\ell}}$, fulfilling the first order differential equation

$$
\nabla^{\left(a_{0}\right.} V^{\left.a_{1} \ldots a_{\ell}\right)}=0 .
$$

The vector space of all Killing tensors of valence $\ell$ will be denoted $\mathcal{K}_{\ell}$.

Since the differential equation (21) is overdetermined, the space $\mathcal{K}_{\ell}$ is finite dimensional. Note that the symmetric product of two Killing tensors is again a Killing tensor, so that $\bigoplus_{\ell \leq 0} \mathcal{K}_{\ell}$ is a commutative graded algebra.

Theorem 4.3. Let $\mathcal{D}$ be a $\ell$-th order commuting symmetry of the Laplace operator. Then the principal symbol $V^{a_{1} \ldots a_{\ell}}$ of $\mathcal{D}$ is a Killing tensor of valence $\ell$.

Proof. When $\mathcal{D}$ is of the form (20), we compute

$$
\Delta \mathcal{D}-\mathcal{D} \Delta=2\left(\nabla^{b} V^{a_{1} \ldots a_{\ell}}\right) \nabla_{b} \nabla_{a_{1}} \ldots \nabla_{a_{\ell}}+" \text { LOTS", }
$$

and the claim follows.

The converse statement is the content of the following Theorem.

Theorem 4.4. There exists a linear map, $V^{a_{1} \ldots a_{\ell}} \mapsto \mathcal{D}^{V}$, from symmetric tensor fields to differential operators, such that the principal symbol of $\mathcal{D}^{V}$ is $V^{a_{1} \ldots a_{\ell}}$ and $\mathcal{D}^{V} \Delta=\Delta \mathcal{D}^{V}$ if $V$ is a Killing tensor.

The proof of the Theorem is postponed to the next section, where the Riemannian prolongation connection for symmetric powers of the adjoint tractor bundle is constructed. This allows to compute explicitly the symmetry operators $\mathcal{D}^{V}$.

Combining both theorems, we deduce a linear bijection between the space of Killing tensors and the space of commuting symmetries of $\Delta$. In particular, the dimension of the vector space of $\ell$-th order symmetry operators is finite, equal to $\operatorname{dim} \mathcal{K}_{0}+\operatorname{dim} \mathcal{K}_{1}+\cdots+\operatorname{dim} \mathcal{K}_{\ell}$.

4.1. Prolongation for Killing tensors. Let $k^{a} \in \mathcal{E}^{a}$ be a Killing vector field. In Remark 3.1, we observed that its prolongation $K=$ $\left(k^{a}, \frac{1}{2} \nabla^{[a} k^{b]}\right) \in \Gamma(\mathcal{A})$ is parallel for the tractor connection. Our aim is to construct analogous prolongation for Killing tensors.

Lemma 4.5. If $k^{a_{1} \ldots a_{\ell}} \in \mathcal{E}^{\left(a_{1} \ldots a_{\ell}\right)}$ is a Killing tensor then

$$
\nabla^{\left(a_{1}\right.} \nabla^{\mid[c} k^{\left.d] \mid a_{2} \ldots a_{\ell}\right)}=-\frac{2(\ell+1)}{n} \mathrm{~J} g^{\left(a_{1} \mid[c\right.} k^{\left.d] \mid a_{2} \ldots a_{\ell}\right)},
$$


where the notation $|\ldots|$ means the enclosed indices $c, d$ are excluded from the symmetrization.

Proof. A straightforward computation.

The prolongation of $k^{a_{1} \ldots a_{\ell}}$ will be a section $K \in \Gamma\left(\otimes^{\ell} \mathcal{A}\right)$. As a first step, we observe the following:

Lemma 4.6. The differential operator

$$
\begin{aligned}
& \Pi: \mathcal{E}^{\left(a_{1} \ldots a_{\ell}\right)} \rightarrow \mathcal{E}^{\left(a_{1} \ldots a_{\ell-1}\right)} \otimes \Gamma(\mathcal{A}), \\
& (\Pi \sigma)^{a_{1} \ldots a_{\ell-1} \mathbf{B}}=\left(\begin{array}{c}
\sigma^{c a_{1} \ldots a_{\ell-1}} \\
\frac{1}{\ell+1} \nabla^{[c} \sigma^{d] a_{1} \ldots a_{\ell-1}}
\end{array}\right) \in \mathcal{E}^{\left(a_{1} \ldots a_{\ell-1}\right)} \otimes \Gamma(\mathcal{A})
\end{aligned}
$$

satisfies, for all $\sigma^{a_{1} \ldots a_{\ell}} \in \mathcal{E}^{\left(a_{1} \ldots a_{\ell}\right)}$,

$$
\nabla^{\left(a_{0}\right.} \sigma^{\left.a_{1} \ldots a_{\ell}\right)}=0 \Longleftrightarrow \nabla^{\left(a_{0}\right.}(\Pi \sigma)^{\left.a_{1} \ldots a_{\ell-1}\right) \mathbf{B}}=0 .
$$

Proof. Using (5), we compute

$$
\nabla^{b}(\Pi \sigma)^{a_{1} \ldots a_{\ell-1} \mathbf{B}}=\left(\begin{array}{c}
\nabla^{b} \sigma^{c a_{1} \ldots a_{\ell-1}}-\frac{2}{\ell+1} \nabla^{[b} \sigma^{c] a_{1} \ldots a_{\ell-1}} \\
\frac{1}{\ell+1} \nabla^{b} \nabla^{[c} \sigma^{d] a_{1} \ldots a_{\ell-1}}+\frac{2}{n} \mathrm{~J} g^{b[c} \sigma^{d] a_{1} \ldots a_{\ell-1}}
\end{array}\right) .
$$

Observe the "top slot" on the right hand side is equal to $\frac{\ell}{\ell+1} \nabla^{b} \sigma^{c a_{1} \ldots a_{\ell-1}}+$ $\frac{1}{\ell+1} \nabla^{c} \sigma^{b a_{1} \ldots a_{\ell-1}}$, which after the symmetrization $\left(b a_{1} \ldots a_{\ell-1}\right)$ yields exactly $\nabla^{(c} \sigma^{\left.b a_{1} \ldots a_{\ell-1}\right)}$. This proves the implication $\Longleftarrow$ of $(\underline{25})$, and also that if $\nabla^{\left(a_{0}\right.} \sigma^{\left.a_{1} \ldots a_{\ell}\right)}=0$ then the "top slot" of $\nabla^{\left(a_{0}\right.}(\Pi \sigma)^{\left.a_{1} \ldots a_{\ell-1}\right)}$ vanishes. Since the bottom slot vanishes by Lemma 4.5 , the implication $\Longrightarrow$ in (25) follows as well.

Considering $\nabla$ in the formula (24) as the coupled Levi-Civita-tractor connection, we obtain the operator $\Pi: \mathcal{E}^{\left(a_{1} \ldots a_{\ell}\right)} \otimes \Gamma(W) \rightarrow \mathcal{E}^{\left(a_{1} \ldots a_{\ell-1}\right)} \otimes$ $\Gamma(\mathcal{A} \otimes W)$, where $W \subseteq(\otimes \mathcal{T}) \otimes\left(\otimes \mathcal{T}^{*}\right)$ is a tractor subbundle. Its iteration

$$
\Pi^{(\ell)}: \mathcal{E}^{\left(a_{1} \ldots a_{\ell}\right)} \rightarrow \Gamma\left(\otimes^{\ell} \mathcal{A}\right)
$$

yields the prolongation for Killing tensors.

Proposition 4.7. Let $\sigma^{a_{1} \ldots a_{\ell}} \in \mathcal{E}^{\left(a_{1} \ldots a_{\ell}\right)}$. The operator $\Pi^{(\ell)}$ satisfies

$$
\nabla^{\left(a_{0}\right.} \sigma^{\left.a_{1} \ldots a_{\ell}\right)}=0 \Longleftrightarrow \nabla\left(\Pi^{(\ell)} \sigma\right)=0 .
$$

Proof. Since the tractor connection is flat, we have the analogue of (25):

$$
\nabla^{\left(a_{0}\right.} \sigma^{\left.a_{1} \ldots a_{\ell}\right) \bullet}=0 \Longleftrightarrow \nabla^{\left(a_{0}\right.}(\Pi \sigma)^{\left.a_{1} \ldots a_{\ell-1}\right) \bullet}=0,
$$

for all $\sigma^{a_{1} \ldots a_{\ell} \bullet} \in \mathcal{E}^{\left(a_{1} \ldots a_{\ell}\right)} \otimes \Gamma(W)$ where $\bullet$ denotes an unspecified tractor index. By iteration, we get then (27). 
The symmetries of the tractor $\Pi^{(\ell)} \sigma$ are best understood in the language of Young diagrams. Putting $\mathcal{T}=\square$, we have $\mathcal{A}=\square$ and we set

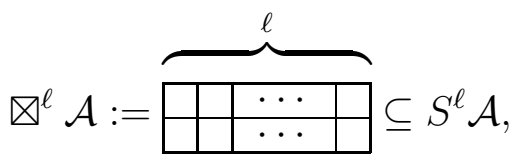

where $S^{\ell} \mathcal{A} \subset \bigotimes^{\ell} \mathcal{A}$ is the subspace of symmetric tensors.

Proposition 4.8. The map $\Pi^{(\ell)}$, defined in (26), is valued in $\Gamma\left(\nabla^{\ell} \mathcal{A}\right)$.

Proof. Let $\sigma^{a_{1} \ldots a_{\ell}} \in \mathcal{E}^{\left(a_{1} \ldots a_{\ell}\right)}$. Using abstract indices $\mathbf{B}_{i}=\left[B_{i}^{1} B_{i}^{2}\right]$, we have $\left(\Pi^{(\ell)} \sigma\right)^{B_{1}^{1} B_{1}^{2} \ldots B_{\ell}^{1} B_{\ell}^{2}} \in \mathcal{E}^{\mathbf{B}_{1} \ldots \mathbf{B}_{\ell}}=\Gamma\left(\otimes^{\ell} \mathcal{A}\right)$.

First, we prove that $\left(\Pi^{(\ell)} \sigma\right)^{\mathbf{B}_{1} \ldots \mathbf{B}_{\ell}}$ is symmetric in indices $\mathbf{B}_{1}, \ldots$, $\mathbf{B}_{\ell}$, i.e $\Pi^{(\ell)} \sigma \in \Gamma\left(S^{\ell} \mathcal{A}\right)$. In fact, it is sufficient to show the symmetry in two neighboring indices $\mathbf{B}_{i}, \mathbf{B}_{i+1}$. To do this, we show that, for all $\sigma^{a_{1} \ldots a_{\ell} \bullet} \in \mathcal{E}^{\left(a_{1} \ldots a_{\ell}\right) \bullet}$ with $\ell \geq 2,\left(\Pi^{(2)} \sigma\right)^{a_{1} \ldots a_{\ell-2} \mathbf{B}_{1} \mathbf{B}_{2} \bullet}$ is symmetric in $\mathbf{B}_{1}$ and $\mathbf{B}_{2}$. This follows from the explicit formula

$$
\begin{aligned}
& \left(\Pi^{(2)} \sigma\right)^{a_{1} \ldots a_{\ell-2} \mathbf{B C} \bullet}=\mathbb{Y}_{b}^{\mathbf{B}} \mathbb{Y}_{c}^{\mathbf{C}} \sigma^{a_{1} \ldots a_{\ell-2} b c \bullet} \\
& \quad+\frac{1}{\ell+1}\left[\mathbb{Y}_{b}^{\mathbf{B}} \mathbb{Z}_{\mathbf{c}}^{\mathbf{C}} \nabla^{c^{1}} \sigma^{a_{1} \ldots a_{\ell-2} b c^{2} \bullet}+\mathbb{Z}_{\mathbf{b}}^{\mathbf{B}_{\mathbf{Y}}} \mathbb{Y}_{c}^{\mathbf{C}} \nabla^{b^{1}} \sigma^{a_{1} \ldots a_{\ell-2} b^{2} c \bullet}\right] \\
& +\frac{1}{\ell} \mathbb{Z}_{\mathbf{b}}^{\mathbf{B}} \mathbb{Z}_{\mathbf{c}}^{\mathbf{C}}\left[\frac{1}{\ell+1} \nabla^{b^{1}} \nabla^{c^{1}} \sigma^{a_{1} \ldots a_{\ell-2} b^{2} c^{2} \bullet}+\frac{2}{n} \mathrm{Jg}^{b^{1} c^{1}} \sigma^{a_{1} \ldots a_{\ell-2} b^{2} c^{2} \bullet}\right]
\end{aligned}
$$

obtained from (24) after a short computation. Here $\mathbf{B}=\left[B^{1} B^{2}\right], \mathbf{C}=$ $\left[C^{1} C^{2}\right], \mathbf{b}=\left[b^{1} b^{2}\right]$ and $\mathbf{c}=\left[c^{1} c^{2}\right]$.

Then, it suffices to prove that $\left(\Pi^{(\ell)} \sigma\right)^{B_{1}^{1} B_{1}^{2} \ldots B_{\ell}^{1} B_{\ell}^{2}} \in \mathcal{E}^{\mathbf{B}_{1} \ldots \mathbf{B}_{\ell}}$ vanishes after skew-symmetrization over any triple of indices $B_{i}^{j}$. Since $\left(\Pi^{(\ell)} \sigma\right)^{\mathbf{B}_{1} \ldots \mathbf{B}_{\ell}}$ is symmetric in tractor form indices $\mathbf{B}_{i}$, it is sufficient to consider only two triples of indices: either $B_{1}^{1}, B_{1}^{2}, B_{2}^{1}$ or $B_{1}^{1}, B_{2}^{1}$, $B_{3}^{1}$. Elementary representation theory shows that the third symmetric power of $\mathcal{A}$ has the decomposition

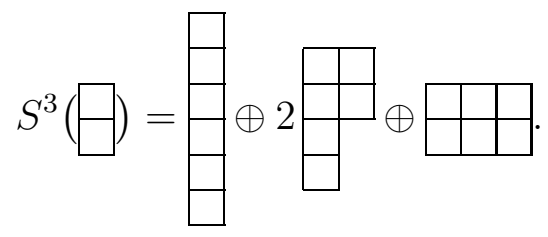

Hence, if we skew over three factors of the standard tractor bundle in $S^{3} \mathcal{E}^{\mathbf{A}}$, the result will be in fact skew symmetric in at least four factors of the standard tractor bundle. As a result, it is sufficient to consider only skew symmetrization over indices $B_{1}^{1}, B_{1}^{2}, B_{2}^{1}$ of $\left(\Pi^{(\ell)} \sigma\right)^{B_{1}^{1} B_{1}^{2} \ldots B_{\ell}^{1} B_{\ell}^{2}}$. It is a straightforward computation to show $\left(\Pi^{(\ell)} \sigma\right)^{\left[B_{1}^{1} B_{1}^{2} B_{2}^{1}\right] B_{2}^{2} \ldots B_{\ell}^{1} B_{\ell}^{2}}=0$, using (29) with $\ell=2$. 
The proposition can be also proved using invariant techniques in parabolic geometries (known as "BGG machinery") applied to the case of projective parabolic geometry. We refer to the next section for further discussion on this relation.

Next, we get the main result of this section

Theorem 4.9. The map $\Pi^{(\ell)}$ induces a bijective correspondence between the space $\mathcal{K}_{\ell}$ of Killing $\ell$-tensors and the space of parallel sections of the tractor bundle $\nabla^{\ell} \mathcal{A}$.

Proof. If $\sigma_{\ell}$ is a Killing $\ell$-tensor, then $\Pi^{(\ell)} \sigma_{\ell}$ is a parallel section of $\nabla^{\ell} \mathcal{A}$, by Propositions 4.7 and 4.8. It remains to prove that, if $F$ is a non-vanishing parallel section of $\nabla^{\ell} \mathcal{A}$, then $F=\Pi^{(\ell)} \sigma_{\ell}$ for some Killing -tensor $\sigma_{\ell}$.

As a section of $\Gamma\left(S^{\ell} \mathcal{A}\right), F$ has the form

$$
F^{\mathbf{A}_{1} \ldots \mathbf{A}_{\ell}}=\sum_{i=0}^{\ell} \mathbb{Y}_{a_{1}}^{\left(\mathbf{A}_{1}\right.} \ldots \mathbb{Y}_{a_{i}}^{\mathbf{A}_{i}} \mathbb{Z}_{\mathbf{c}_{i+1}}^{\mathbf{A}_{i+1}} \ldots \mathbb{Z}_{\mathbf{c}_{\ell}}^{\left.\mathbf{A}_{\ell}\right)}\left(\sigma_{i}\right)^{a_{1} \ldots a_{i} \mathbf{c}_{i+1} \ldots \mathbf{c}_{\ell}}
$$

where $\left(\mathbf{A}_{1} \ldots \mathbf{A}_{\ell}\right)$ denotes the symmetrization over the form tractor indices (and not over the standard tractor indices). Here $\left(\sigma_{i}\right)^{a_{1} \ldots a_{i} \mathbf{c}_{i+1} \ldots \mathbf{c}_{\ell}} \in$ $\mathcal{E}^{a_{1} \ldots a_{i} \mathbf{c}_{i+1} \ldots \mathbf{c}_{\ell}}$ where $a_{i}$ are indices of the tangent bundle whereas $\mathbf{c}_{i}=$ $\left[c_{i}^{1} c_{i}^{2}\right]$ are form indices. Since $F \in \Gamma\left(\nabla^{\ell} \mathcal{A}\right)$, the skew symmetrization over any triple of indices of $\left(\sigma_{i}\right)^{a_{1} \ldots a_{i}\left[c_{i+1}^{1} c_{i+1}^{2}\right] \ldots\left[c_{\ell}^{1} c_{\ell}^{2}\right]}$ vanishes.

First, we show that $\sigma_{\ell}=0$ implies $F=0$. To do that, we assume $\sigma_{i_{0}+1}=\ldots=\sigma_{\ell}=0$ and prove $\sigma_{i_{0}}=0$, with $0 \leq i_{0}<\ell$. The tractor form $\nabla^{b} F^{\mathbf{A}_{1} \ldots \mathbf{A}_{\ell}}$ can be written in the form (31), and it follows from (14) that

$$
\begin{aligned}
\nabla^{b} F^{\mathbf{A}_{1} \ldots \mathbf{A}_{\ell}}= & 2\left(\ell-i_{0}\right) \mathbb{Y}_{a_{1}}^{\left(\mathbf{A}_{1}\right.} \ldots \mathbb{Y}_{a_{i_{0}+1}}^{\mathbf{A}_{i_{0}+1}} \mathbb{Z}_{\mathbf{c}_{i_{0}+2}}^{\mathbf{A}_{i_{0}+2}} \ldots \mathbb{Z}_{\mathbf{c}_{\ell}}^{\left.\mathbf{A}_{\ell}\right)}\left(\sigma_{i_{0}}\right)^{\left(a_{1} \ldots a_{i_{0}} a_{i_{0}+1}\right) b \mathbf{c}_{i_{0}+2} \ldots \mathbf{c}_{\ell}} \\
& + \text { terms with at most } i_{0} \text { of } \mathbb{Y} \text { 's. }
\end{aligned}
$$

Thus $\left(\sigma_{i_{0}}\right)^{\left(a_{1} \ldots a_{i_{0}} a_{i_{0}+1}\right) b \mathbf{c}_{i_{0}+2} \ldots \mathbf{c}_{\ell}}=0$. On the other hand, symmetries of $F$ imply that symmetries of $\left(\sigma_{i_{0}}\right)^{a_{1} \ldots a_{i_{0}} \mathbf{c}_{i_{0}+1 \ldots \mathbf{c}_{\ell}}}$ correspond to the Young

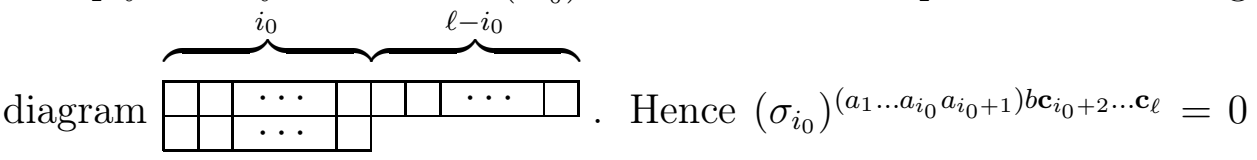

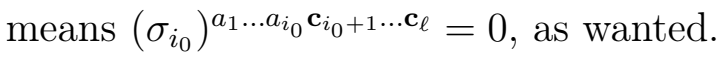

Next we need to show that the tensor field $\left(\sigma_{\ell}\right)^{a_{1} \ldots a_{\ell}}$ is Killing. Similarly as above, computing the $\mathbb{Y}_{a_{1}}^{\left(\mathbf{A}_{1}\right.} \ldots \mathbb{Y}_{\left.a_{\ell}\right)}^{\left.\mathbf{A}_{\ell}\right)}$-summand of $\nabla^{b} F^{\mathbf{A}_{1} \ldots \mathbf{A}_{\ell}}$ (which is zero), one easily concludes that $\nabla^{(b}\left(\sigma_{\ell}\right)^{\left.a_{1} \ldots a_{\ell}\right)}=0$. Details are left to the reader. Finally, since the difference $F-\Pi^{(\ell)} \sigma_{\ell} \in \Gamma\left(\nabla^{\ell} \mathcal{A}\right)$ is parallel and the $\mathbb{Y}_{a_{1}}^{\left(\mathbf{A}_{1}\right.} \ldots \mathbb{Y}_{a_{\ell}}^{\left.\mathbf{A}_{\ell}\right)}$-summand of $F-\Pi^{(\ell)} \sigma_{\ell}$ vanishes, it follows from the first part of the proof that $F-\Pi^{(\ell)} \sigma_{\ell}=0$.

4.2. Construction of commuting symmetries. Let $V^{a_{1} \ldots a_{\ell}} \in \mathcal{E}^{\left(a_{1} \ldots a_{\ell}\right)}$ be a symmetric tensor and $\Pi^{(\ell)}$ the map defined in (26) . We define the 
differential operator $\mathcal{D}^{V}$ of order $\ell$ by

$$
\mathcal{D}^{V}:=\left\langle\Pi^{(\ell)} V, \mathbb{D}^{(\ell)}\right\rangle: \Gamma(U) \rightarrow \Gamma(U),
$$

where $\mathbb{D}^{(\ell)}: \Gamma(U) \rightarrow \bigotimes^{\ell} \Gamma\left(\mathcal{A}^{*}\right) \otimes \Gamma(U)$ is the $\ell$ th iteration of the operator (16).

Lemma 4.10. The differential operator $\mathcal{D}^{V}$ has principal symbol $V^{a_{1} \ldots a_{\ell}}$.

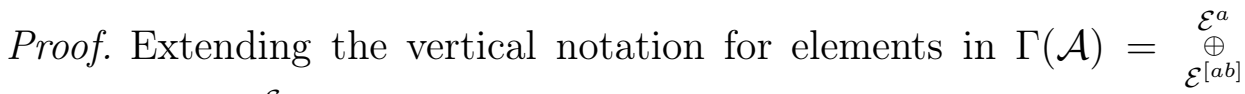

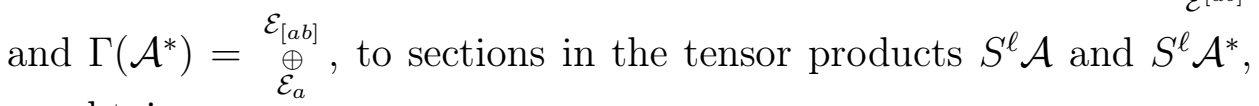
we obtain

$$
\Pi^{(\ell)} V=\begin{gathered}
V^{a_{1} \ldots a_{\ell}} \\
\oplus \\
\vdots
\end{gathered} \quad \begin{gathered}
\mathcal{E}^{a_{1} \ldots a_{\ell}} \\
\vdots
\end{gathered} \quad \text { and } \mathbb{D}^{(\ell)} u=\begin{gathered}
\vdots \\
\nabla_{a_{1}} \ldots \nabla_{a_{\ell}} u
\end{gathered} \mathcal{E}_{a_{1} \ldots a_{\ell}} \otimes \Gamma(U) .
$$

Thus the contraction $\left\langle\Pi^{(\ell)} V, \mathbb{D}^{(\ell)} u\right\rangle$ has the leading term $V^{a_{1} \ldots a_{\ell}} \nabla_{a_{1}} \ldots \nabla_{a_{\ell}} u$.

We consider a Riemannian invariant linear differential operator $F$ : $\Gamma(U) \rightarrow \Gamma(U)$, acting on a tensor bundle $U$.

Theorem 4.11. Let $k^{a_{1} \ldots a_{\ell}} \in \mathcal{E}^{\left(a_{1} \ldots a_{\ell}\right)}$ be a Killing tensor. Then the differential operator $\mathcal{D}^{k}$ is a commuting symmetry of $F$ with principal symbol $k^{a_{1} \ldots a_{\ell}}$.

Proof. By Lemma 4.10, $\mathcal{D}^{k}$ has principal symbol $k^{a_{1} \ldots a_{\ell}}$.

Let $u \in \Gamma(U)$ and $K:=\Pi^{(\ell)} k \in \Gamma\left(\otimes^{\ell} \mathcal{A}\right)$ be the prolongation of the Killing $\ell$-tensor $k$. Then we get

$$
F \mathcal{D}^{k} u=F\left\langle K, \mathbb{D}^{(\ell)} u\right\rangle=\left\langle K, F^{\nabla} \mathbb{D}^{(\ell)} u\right\rangle=\left\langle K, \mathbb{D}^{(\ell)} F u\right\rangle=\mathcal{D}^{k} F u,
$$

where we have used Proposition 4.7 (which implies $\nabla K=0$ ) in the second equality and Corollary 3.3 in the third equality. Recall the operator $F^{\nabla}$ is given by the same formula as $F$ but $\nabla$ is interpreted as the coupled Levi-Civita-tractor connection in $F^{\nabla}$.

Corollary 4.12. Let us assume $k^{a_{1} \ldots a_{\ell}} \in \mathcal{E}^{\left(a_{1} \ldots a_{\ell}\right)}$ is a Killing tensor. Then $\mathcal{D}^{k}$ is a commuting symmetry of the Laplacian $\Delta: \mathcal{E} \rightarrow \mathcal{E}$ with principal symbol $k^{a_{1} \ldots a_{\ell}}$.

4.3. Algebra structure on the space of commuting symmetries of the Laplace operator $\Delta: \mathcal{E} \rightarrow \mathcal{E}$. Let $\mathcal{B}$ be the algebra of commuting symmetries of $\Delta$. The Theorems 4.3 and 4.4 allow us to identify the vector space of commuting symmetries of $\Delta$,

$$
\mathcal{B} \simeq \bigoplus_{\ell=0}^{\infty} \mathcal{K}_{\ell}
$$

In order to study the algebra structure on $\mathcal{B}$, some basic notation is needed. Depending on the curvature, the Lie group of isometries 
is $G=S O(p+1, q), G=S O(p, q+1)$ or $G=E(p, q)$. For all possibilities, we denote by $\mathfrak{g}=\operatorname{Lie}(G)$ the Lie algebra of isometries and use the identifications $\mathfrak{s o}(p+1, q) \simeq \wedge^{2} \mathbb{R}^{p+1, q}, \mathfrak{s o}(p, q+1) \simeq \wedge^{2} \mathbb{R}^{p, q+1}$, and $\operatorname{Lie}(E(p, q)) \simeq \wedge^{2}\left(\mathbb{R}^{p, q} \rightarrow \mathbb{R}\right)$. In the last case, the identification is deduced from the representation of $E(p, q)$ on $\mathbb{R}^{p, q} \oplus \mathbb{R}$, induced by the standard group morphism $\mathrm{GL}(n, \mathbb{R}) \ltimes \mathbb{R}^{n} \rightarrow \mathrm{GL}(n+1, \mathbb{R})$.

The space of parallel sections of $\mathcal{A}$ is isomorphic to $\mathfrak{g}$ and it is easy to verify that the Lie bracket on $\mathfrak{g}$ is isomorphic to the bracket (8) . Via the induced identification of the symmetric product $S^{\ell} \mathfrak{g}$ with parallel sections of $S^{\ell} \mathcal{A}$, we define the subspace $\nabla^{\ell} \mathfrak{g} \subseteq S^{\ell} \mathfrak{g}$ as follows

$$
\nabla^{\ell} \mathfrak{g} \cong\left\{\text { parallel sections of } \nabla^{\ell} \mathcal{A}\right\},
$$

where $\nabla^{\ell} \mathcal{A}$ is defined in (28). From Theorem 4.9, we deduce that $\nabla^{\ell} \mathfrak{g}$ is isomorphic, as $\mathfrak{g}$-module, to the space $\mathcal{K}_{\ell}$ of Killing $\ell$-tensors. Hence $\mathcal{B} \simeq \bigoplus_{\ell=0}^{\infty} \nabla^{\ell} \mathfrak{g}$ as $\mathfrak{g}$-module.

Theorem 4.13. The symmetry algebra $\mathcal{B}$ is isomorphic to the tensor algebra

$$
\bigoplus_{i=0}^{\infty} \otimes^{i} \mathfrak{g}
$$

modulo the two-sided ideal $\mathcal{I}$, generated by

$$
V \otimes W-V \otimes W-\frac{1}{2}[V, W], \quad V, W \in \mathfrak{g} .
$$

Proof. First we compute the compositions $\mathcal{D}^{k} \mathcal{D}^{\check{k}}$, where $k^{a}, \check{k}^{a} \in \Gamma(T M)$ are Killing vector fields. Put $K=\Pi k^{a}$ and $\check{K}=\Pi \check{k}^{a}$ where $K, \check{K} \in \mathfrak{g}$. Since $\nabla \check{K}=0$, the definition (19) of $\mathbb{D}_{\mathbf{B}}$ acting on functions yields

$$
\begin{aligned}
\mathcal{D}^{k} \mathcal{D}^{\check{k}} & =K^{\mathbf{B}} \mathbb{D}_{\mathbf{B}} \check{K}^{\mathbf{C}} \mathbb{D}_{\mathbf{C}} \\
& =\left[\frac{1}{2}\left(K^{\mathbf{B}} \check{K}^{\mathbf{C}}+K^{\mathbf{C}} \check{K}^{\mathbf{B}}\right)+\frac{1}{2}\left(K^{\mathbf{B}} \check{K}^{\mathbf{C}}-K^{\mathbf{C}} \check{K}^{\mathbf{B}}\right)\right] \mathbb{D}_{\mathbf{B}} \mathbb{D}_{\mathbf{C}} \\
& =(K \otimes \check{K})^{\mathbf{B C}} \mathbb{D}_{\mathbf{B}} \mathbb{D}_{\mathbf{C}}+\frac{1}{2}[K, \check{K}]^{\mathbf{B}} \mathbb{D}_{\mathbf{B}} .
\end{aligned}
$$

In the last equality, to deal with the symmetrized term, we use the decomposition

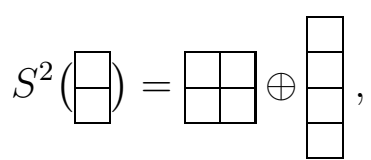

and the identity $\mathbb{D}_{\left[B^{1} B^{2}\right.} \mathbb{D}_{\left.C^{1} C^{2}\right]}=0$, which can be easily verified. To deal with the skew-symmetrized term, we use (8) .

The computation of $\mathcal{D}^{k} \mathcal{D}^{\check{k}}$ shows that all elements of the form (35) are in the ideal. Since there is a vector space isomorphism

$$
\left(\bigoplus_{\ell=0}^{\infty} \otimes^{\ell} \mathfrak{g}\right) / \mathcal{I} \cong \bigoplus_{\ell=0}^{\infty} \nabla^{\ell} \mathfrak{g}
$$


it remains to show that elements in $\nabla^{\ell} \mathfrak{g} \cong \mathcal{K}_{\ell}$ indeed give rise to nonzero $\ell$ th order symmetries. This follows from Corollary 4.12. The proof is complete.

The passage from the tensor algebra to the universal enveloping algebra $\mathcal{U}(\mathfrak{g})$ means to substitute $V \otimes W=\frac{1}{2}(V \otimes W+W \otimes V)-\frac{1}{2}(V \otimes$ $W-W \otimes V)$ and quotient through the two-sided ideal generated by $V \otimes W-W \otimes V=[V, W], V, W \in \mathfrak{g}$. Accordingly, we get

Corollary 4.14. The symmetry algebra $\mathcal{B}$ is isomorphic to the universal enveloping algebra $\mathcal{U}(\mathfrak{g})$ modulo the two-sided ideal generated by

$$
V \otimes W+W \otimes V-2 V \otimes W, \quad V, W \in \mathfrak{g} .
$$

4.4. Examples of commuting symmetries. The recursion tractor formula (32) for commuting symmetries $\mathcal{D}^{k}$ can be transformed into an explicit formula for $\mathcal{D}^{k}$, expressed in terms of the Levi-Civita connection $\nabla$ and the curvature J, by (25) and (19). In what follows we compute the explicit commuting symmetries up to order 3 acting on $\mathcal{E}$.

We use tractor form indices $\mathbf{A}=\left[A^{1} A^{2}\right], \mathbf{B}=\left[B^{1} B^{2}\right], \mathbf{C}=\left[C^{1} C^{2}\right]$ and form indices $\mathbf{a}=\left[a^{1} a^{2}\right], \mathbf{b}=\left[b^{1} b^{2}\right], \mathbf{c}=\left[c^{1} c^{2}\right]$.

For a Killing vector field $k^{a} \in \mathcal{E}^{a}$, we have

$$
K^{\mathbf{A}}=(\Pi k)^{\mathbf{A}}=\mathbb{Y}_{a}^{\mathbf{A}} k^{a}+\frac{1}{2} \mathbb{Z}_{\mathbf{a}}^{\mathbf{A}} \nabla^{\left[a^{1}\right.} k^{\left.a^{2}\right]}, \quad \mathbb{D}_{\mathbf{A}} f=2 \mathbb{Y}_{\mathbf{A}}^{a} \nabla_{a} f
$$

Hence, the symmetry $\mathcal{D}^{k} f=K^{\mathbf{A}} \mathbb{D}_{\mathbf{A}} f=k^{a} \nabla_{a} f$ coincides with the Lie derivative along the Killing vector field $k^{a}$.

For a Killing 2-tensor $k^{b c} \in \mathcal{E}^{(b c)}$, we get from (29)

$$
\begin{aligned}
K^{\mathbf{A B}}= & \left(\Pi^{(2)} k\right)^{\mathbf{B C}}=\mathbb{Y}_{b}^{\mathbf{B}} \mathbb{Y}_{c}^{\mathbf{C}} k^{b c}+\frac{1}{3}\left(\mathbb{Y}_{b}^{\mathbf{B}} \mathbb{Z}_{\mathbf{c}}^{\mathbf{C}}+\mathbb{Y}_{b}^{\mathbf{C}} \mathbb{Z}_{\mathbf{c}}^{\mathbf{B}}\right) \nabla^{c^{1}} k^{c^{2} b} \\
& +\frac{1}{2} \mathbb{Z}_{\mathbf{b}}^{\mathbf{B}} \mathbb{Z}_{\mathbf{c}}^{\mathbf{C}}\left[\frac{1}{3} \nabla^{b^{1}} \nabla^{c^{1}} k^{b^{2} c^{2}}+\frac{2}{n} \mathrm{Jg}^{b^{1} c^{1}} k^{b^{2} c^{2}}\right] .
\end{aligned}
$$

Since $\mathbb{D}_{\mathbf{B}} \mathbb{D}_{\mathbf{C}} f=4 \mathbb{Y}_{\mathbf{B}}^{b} \mathbb{Z}_{\mathbf{C}}^{\mathbf{c}} g_{b c^{0}} \nabla_{c^{1}} f+4 \mathbb{Y}_{\mathbf{B}}^{b} \mathbb{Y}_{\mathbf{C}}^{c} \nabla_{b} \nabla_{c} f$ by (19), we obtain

$$
\mathcal{D}^{k}=K^{\mathbf{B C}} \mathbb{D}_{\mathbf{B}} \mathbb{D}_{\mathbf{C}} f=k^{b c} \nabla_{b} \nabla_{c} f+\left(\nabla_{r} k^{r c}\right) \nabla_{c} f .
$$

Note that $K^{\mathbf{B C}} h_{\mathbf{B C}}$ is a constant, and using $\nabla^{a} k_{r}^{r}=-2 \nabla_{r} k^{a r}$ (which follows from $3 g_{b c} \nabla^{(a} k^{b c)}=\nabla^{a} k_{r}^{r}+2 \nabla_{r} k^{a r}=0$ ), a short computation reveals its value

$$
K^{\mathbf{B C}} h_{\mathbf{B C}}=\frac{1}{4}\left[-\nabla_{r} \nabla_{s} \sigma^{r s}+\frac{2(n+1)}{n} \mathrm{~J}_{r}^{r}\right] .
$$

Thus the modification of $\mathcal{D}^{k}$ by any multiple of $\nabla_{r} \nabla_{s} k^{r s}-\frac{2(n+1)}{n} \mathrm{~J} k^{r}{ }_{r}$ is again a symmetry of $\Delta$. This means there is no unique formula, written in terms of $k^{a b}$, for a symmetry. This is in contrast with the case of conformal symmetries [8].

Now we consider Killing 3-tensors $k^{a b c} \in \mathcal{E}^{(a b c)}$. Then

$$
g_{c d} \nabla^{(a} k^{b c d)}=3 \nabla_{r} k^{r a b}+3 \nabla^{a} k_{r}^{b r}=0,
$$


and applying $\nabla_{a}$ we obtain $\nabla_{r} \nabla_{s} k^{r s a}+\Delta k_{r}^{a r}=0$. Summarizing, we obtain

$$
\nabla_{r} k^{r a b}=-\nabla^{a} k_{r}^{b r}, \quad \nabla_{r} \nabla_{s} k^{r s a}=-\Delta k_{r}^{a r} \quad \text { and } \quad \nabla_{r} k_{s}^{r s}=0,
$$

where the last equality is the trace of $\nabla_{r} k^{r a b}+\nabla^{a} k^{b r}{ }_{r}=0$. Now computing $\Pi^{(3)} k$ (which requires to apply $\Pi$ in (24) to (29)) results in

$$
\begin{aligned}
& K^{\mathbf{A B C}}=\left(\Pi^{(3)} k\right)^{\mathbf{A B C}}=\mathbb{Y}_{a}^{\mathbf{A}} \mathbb{Y}_{b}^{\mathbf{B}} \mathbb{Y}_{c}^{\mathbf{C}} k^{a b c} \\
& +\frac{1}{4}\left(\mathbb{Y}_{a}^{\mathbf{A}} \mathbb{Y}_{b}^{\mathbf{B}} \mathbb{Z}_{\mathbf{c}}^{\mathbf{C}}+\mathbb{Y}_{a}^{\mathbf{C}} \mathbb{Y}_{b}^{\mathbf{A}} \mathbb{Z}_{\mathbf{c}}^{\mathbf{B}}+\mathbb{Y}_{a}^{\mathbf{B}} \mathbb{Y}_{b}^{\mathbf{C}} \mathbb{Z}_{\mathbf{c}}^{\mathbf{A}}\right) \nabla^{c^{1}} k^{c^{2} a b} \\
& +\frac{1}{3}\left(\mathbb{Y}_{a}^{\mathbf{A}} \mathbb{Z}_{\mathbf{b}}^{\mathbf{B}} \mathbb{Z}_{\mathbf{c}}^{\mathbf{C}}+\mathbb{Y}_{a}^{\mathbf{C}} \mathbb{Z}_{\mathbf{b}}^{\mathbf{A}} \mathbb{Z}_{\mathbf{c}}^{\mathbf{B}}+\mathbb{Y}_{a}^{\mathbf{B}} \mathbb{Z}_{\mathbf{b}}^{\mathbf{C}} \mathbb{Z}_{\mathbf{c}}^{\mathbf{A}}\right)\left[\frac{1}{4} \nabla^{b^{1}} \nabla^{c^{1}} k^{b^{2} c^{2} a}+\frac{2}{n} \mathrm{Jg}^{b^{1} c^{1}} k^{b^{2} c^{2} a}\right] \\
& +\mathbb{Z}_{\mathbf{a}}^{\mathbf{A}} \mathbb{Z}_{\mathbf{b}}^{\mathbf{B}} \mathbb{Z}_{\mathbf{c}}^{\mathbf{C}} \psi_{\mathbf{a b c}}
\end{aligned}
$$

for some $\psi_{\mathbf{a b c}}$ which we do not need to compute. Furthermore,

$$
\begin{aligned}
\mathbb{D}_{\mathbf{A}} \mathbb{D}_{\mathbf{B}} \mathbb{D}_{\mathbf{C}} f=8 \mathbb{Y}_{\mathbf{A}}^{a} \mathbb{Z}_{\mathbf{B}}^{\mathbf{b}} \mathbb{Z}_{\mathbf{C}}^{\mathbf{c}} g_{a b^{1}} g_{b^{2} c^{1}} \nabla_{c^{2}} f \\
\quad+16 \mathbb{Y}_{\mathbf{A}}^{a} \mathbb{Y}_{\mathbf{B}}^{b} \mathbb{Z}_{\mathbf{C}}^{\mathbf{c}} g_{c^{1}(a} \nabla_{b)} \nabla_{c^{2}} f+8 \mathbb{Y}_{\mathbf{A}}^{a} \mathbb{Z}_{\mathbf{B}}^{\mathbf{b}} \mathbb{Y}_{\mathbf{C}}^{c} g_{a b^{1}} \nabla_{c} \nabla_{b^{2}} f \\
\quad+8 \mathbb{Y}_{\mathbf{A}}^{a} \mathbb{Y}_{\mathbf{B}}^{b} \mathbb{Y}_{\mathbf{C}}^{c}\left[\nabla_{a} \nabla_{b} \nabla_{c} f-\frac{4}{n} \mathrm{~J} g_{b[a} \nabla_{c]} f\right]
\end{aligned}
$$

by (19). Combining the two previous displays yields

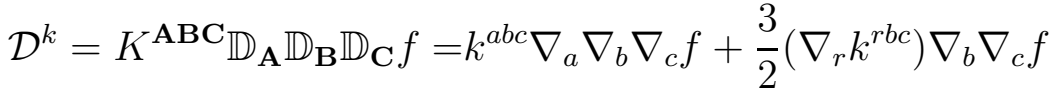

$$
\begin{aligned}
& +\frac{1}{4}\left(\nabla_{r} \nabla_{s} k^{r s c}\right) \nabla_{c} f-\frac{n-1}{2 n} \mathrm{~J}^{c r}{ }_{r} \nabla_{c} f .
\end{aligned}
$$

By construction, the vector field $\left(\Pi^{(2)} k\right)^{a \mathbf{B C}} h_{\mathbf{B C}}$ is Killing. Using (29), (15) and (39), one easily computes

$$
\left(\Pi^{(2)} k\right)^{a \mathbf{B C}} h_{\mathbf{B C}}=-\frac{1}{12}\left[\nabla_{r} \nabla_{s} k^{r s a}-\frac{4(n+2)}{n} \mathrm{Jk}_{r}^{a r}\right] .
$$

Thus the symmetry $\mathcal{D}^{k}$ can be modified by a multiple of the operator $\left[\nabla_{r} \nabla_{s} k^{r s a}-\frac{4(n+2)}{n} \mathrm{~J} k_{r}^{a r}\right] \nabla_{a} f$.

Remark 4.15. Let $\ell \in \mathbb{N}$ and $k \in \mathcal{K}_{\ell}$. If the curvature of the metric $g$ vanishes, i.e. $M$ is locally isomorphic to the pseudo-Euclidean space $\mathbb{R}^{p, q}$, a straightforward computation shows that

$$
\mathcal{D}^{k}=\sum_{i=0}^{\ell} \frac{1}{2^{i}}\left(\begin{array}{l}
\ell \\
i
\end{array}\right)\left(\nabla_{a_{1}} \cdots \nabla_{a_{i}} k^{a_{1} \cdots a_{\ell}}\right) \nabla_{a_{i+1}} \cdots \nabla_{a_{\ell}}
$$

is a commuting symmetry of $\Delta$. This can also be deduced from properties of the Weyl quantization of $T^{*} \mathbb{R}^{p, q}$. Namely, $\mathcal{D}^{k}$ coincides with the Weyl quantization of $k$, and the symplectic equivariance of the Weyl quantization (see, e.g., [10]) yields the equalities $\left[\Delta, \mathcal{D}^{k}\right]=[g, k]_{S}=0$. Here, $[\cdot, \cdot]_{S}$ denotes the Schouten bracket of symmetric tensors and $[g, k]_{S}=0$ is equivalent to the Killing equation. 
Remark 4.16. Let $\ell \in \mathbb{N}$ and $k \in \mathcal{K}_{\ell}$. If $k$ is trace-free, then a straightforward computation shows that

$$
\mathcal{D}^{k}=k^{a_{1} \cdots a_{\ell}} \nabla_{a_{1}} \cdots \nabla_{a_{\ell}}
$$

is a commuting symmetry of $\Delta$. This can also be deduced from the work of Eastwood [8]. Out of conformal Killing tensors $V$, he explicitly builds conformal symmetries of the Laplacian, i.e., differential operators $\mathcal{D}_{1}^{V}$ and $\mathcal{D}_{2}^{V}$ with principal symbol $V$ such that $\mathcal{D}_{2}^{V} \Delta=\Delta \mathcal{D}_{1}^{V}$. The lower order terms involve divergences of $V$ and contractions of $V$ with the trace-free Ricci tensor. On a space of constant curvature, with $V=k$ a trace-free Killing tensor, both vanish and we get $\mathcal{D}_{1}^{k}=\mathcal{D}_{2}^{k}=\mathcal{D}^{k}$.

\section{Riemannian GeOMETRY Via PROJECTIVE GEOMETRY}

Overdetermined equations for Killing tensors are projectively invariant [9], so it is natural to consider their prolongation within the framework of projective geometry. As this is an example of a parabolic geometry, we can employ the general invariant theory for this class of structures, [5]. We shall observe that several results obtain in the previous section then follow immediately.

Recall we are interested in manifolds of constant curvature. These are conformally flat and thus projectively flat as well (see (42) below). That is, we will consider locally flat projective structures.

5.1. Tractor calculus in projective geometry. We shall briefly recall invariant calculus on projective manifolds, see [1] for more details. A projective structure on a manifold $M$ is given by a class $[\nabla]$ of special affine connections with the same geodesics as unparametrized curves ("special" means that there is a parallel volume form for every connection in $[\nabla]$.) These connections are parametrized by nowhere vanishing sections of projective density bundles $\mathcal{E}(1)$. We shall also assume orientability, characterized by a compatible volume form $\epsilon_{a^{1} \ldots a^{n}} \in$ $\mathcal{E}_{\left[a^{1} \ldots a^{n}\right]}(n+1) \cong \mathcal{E}$, parallel for every affine connection in $[\nabla]$. The decomposition of the curvature of $\nabla$ is

$$
R_{a b d}^{c}=\bar{C}_{a b d}^{c}+2 \delta_{[a}^{c} \overline{\mathrm{P}}_{b] d},
$$

where $\overline{\mathrm{P}}_{a b}$ is the projective Schouten tensor and $\bar{C}_{a b}{ }^{c}{ }_{d}=C_{a b}{ }^{c}$. (That is, conformal and projective Weyl tensors coincide.) Note that for the Levi-Civita connection $\nabla$ of an Einstein metric $g$, the curvature is also of the form (11) and the relation between projective and Riemannian Schouten tensors is $\overline{\mathrm{P}}_{a b}=2 \mathrm{P}_{a b},[11$.

We define the standard tractor bundle and its dual by their spaces of sections $\overline{\mathcal{E}}^{A}$ and $\overline{\mathcal{E}}_{A}$, respectively, as

$$
\overline{\mathcal{E}}^{A}=\begin{gathered}
\mathcal{E}^{a}(-1) \\
\mathcal{E}(-1)
\end{gathered} \quad \text { and } \quad \overline{\mathcal{E}}_{A}=\begin{gathered}
\mathcal{E}(1) \\
\uparrow \\
\mathcal{E}_{a}(1)
\end{gathered},
$$


see [1] for the meaning of the semi-direct product $\uparrow$. The choice of a connection in the class $[\nabla]$ turns the previous display into the direct sum decomposition. These bundles are equipped with the projectively invariant tractor connection which we denote by $\bar{\nabla}$. Choosing $\nabla$ in the projective class, $\bar{\nabla}$ is explicitly given by the formulas

$$
\bar{\nabla}_{a}\left(\begin{array}{c}
\nu^{b} \\
\rho
\end{array}\right)=\left(\begin{array}{c}
\nabla_{a} \nu^{b}+\delta_{a}^{b} \rho \\
\nabla_{a} \rho-\overline{\mathrm{P}}_{a b} \nu^{b}
\end{array}\right) \quad \text { and } \quad \bar{\nabla}_{a}\left(\begin{array}{c}
\sigma \\
\mu_{b}
\end{array}\right)=\left(\begin{array}{c}
\nabla_{a} \sigma-\mu_{a} \\
\nabla_{a} \mu_{b}+\overline{\mathrm{P}}_{a b} \sigma
\end{array}\right)
$$

see [1 for details. Here $\nu^{a} \in \mathcal{E}^{a}(-1), \rho \in \mathcal{E}(-1), \sigma \in \mathcal{E}(1)$ and $\mu_{a} \in \mathcal{E}_{a}(1)$. We extend the connection $\bar{\nabla}$ to the tensor products of $\overline{\mathcal{E}}^{A}$ by the Leibniz rule. Also note that the structure of the tractor bundle $\overline{\mathcal{E}}^{[A B]}$ and of its dual is given by

$$
\overline{\mathcal{E}}^{[A B]}=\begin{aligned}
& \mathcal{E}^{[a b]}(-2) \\
& \mathcal{E}^{a}(-2)
\end{aligned} \quad \text { and } \quad \overline{\mathcal{E}}_{[A B]}=\begin{gathered}
\mathcal{E}_{a}(2) \\
\uparrow \\
\mathcal{E}_{[a b]}(2)
\end{gathered}
$$

In what follows, we shall use the tractor bundle

$$
\begin{aligned}
& \mathcal{E}^{a}
\end{aligned}
$$

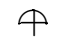

$$
\begin{aligned}
& \overline{\mathcal{E}}_{A}^{B}=\overline{\mathcal{E}}_{A} \otimes \overline{\mathcal{E}}^{B}=\mathcal{E}_{a}^{b} \oplus \mathcal{E}, \\
& \uparrow \\
& \mathcal{E}_{a}
\end{aligned}
$$

where the trace-free part of $\overline{\mathcal{E}}_{A}^{B}$ is isomorphic to the projective adjoint tractor bundle. Analogously to (16), we define the projectively invariant differential operator

$$
\overline{\mathbb{D}}_{A}^{B}: \mathcal{E}_{b_{1} \ldots b_{s}}(w) \otimes \overline{\mathcal{E}}_{C \ldots D}^{E \ldots F} \rightarrow \mathcal{E}_{b_{1} \ldots b_{s}}(w) \otimes \overline{\mathcal{E}}_{A}^{B} \otimes \overline{\mathcal{E}}_{C \ldots D}^{E \ldots F},
$$

as follows. Acting on $f \in \mathcal{E}(w)$ and $\varphi_{a} \in \mathcal{E}_{a}, \overline{\mathbb{D}}_{A}{ }^{B}$ is given by

$$
\overline{\mathbb{D}}_{A}^{B} f=\left(\begin{array}{c}
0 \\
0 \mid w f \\
\nabla_{a} f
\end{array}\right), \quad \overline{\mathbb{D}}_{A}{ }^{B} \varphi_{c}=\left(\begin{array}{c}
0 \\
\delta_{c}^{b} \varphi_{a} \mid-\varphi_{c} \\
\nabla_{a} \varphi_{c}
\end{array}\right),
$$

for an affine connection $\nabla$ in the projective class. The formula for $\overline{\mathbb{D}}_{A}{ }^{B} f$ for $f \in \overline{\mathcal{E}}_{C \ldots D}{ }^{E \ldots F}$ is formally the same as for $f \in \mathcal{E}(0)$ where we interpret $\nabla$ as the coupled affine-tractor connection. Then we extend $\overline{\mathbb{D}}_{A}^{B}$ to the general case by the Leibniz rule.

Henceforth, we assume the manifold $M$ is projectively flat, i.e., the projective Weyl tensor vanishes. This in particular means that the tractor connection $\bar{\nabla}$ is flat.

Let $F: \Gamma\left(U_{1}\right) \rightarrow \Gamma\left(U_{2}\right)$ be a projectively invariant linear differential operator, acting between tensor bundles $U_{1}, U_{2}$. Then, $F$ can be written in terms of an affine connection $\nabla$. Regarding $\nabla$ in the formula for $F$ as the coupled affine-tractor connection, we obtain the operator 
$F^{\bar{\nabla}}: \Gamma\left(\mathcal{A}^{*} \otimes U_{1}\right) \rightarrow \Gamma\left(\mathcal{A}^{*} \otimes U_{2}\right)$. Adapting the proof of Theorem 3.2 to the projective setting, we obtain the analogue of Corollary 3.3 .

Theorem 5.1. Let $F: \Gamma\left(U_{1}\right) \rightarrow \Gamma\left(U_{2}\right)$ be a projectively invariant linear differential operator over a projectively flat manifold. Then $\overline{\mathbb{D}}_{A}{ }^{B}$ commutes with $F$, i.e.

$$
\overline{\mathbb{D}} \circ F=F^{\bar{\nabla}} \circ \overline{\mathbb{D}}: \Gamma\left(U_{1}\right) \rightarrow \overline{\mathcal{E}}_{A}^{B} \otimes \Gamma\left(U_{2}\right) .
$$

As an example, consider the projectively invariant differential operator

$$
\nabla_{(a} \nabla_{b)}+\overline{\mathrm{P}}_{a b}: \mathcal{E}(1) \rightarrow \mathcal{E}_{(a b)}(1)
$$

see e.g. [4]. The projective invariance and Theorem 5.1 imply $(49) \overline{\mathbb{D}}_{A_{1}}^{B_{1}} \ldots \overline{\mathbb{D}}_{A_{\ell}}^{B_{\ell}}\left(\nabla_{(a} \nabla_{b)}+\overline{\mathrm{P}}_{a b}\right)=\left(\nabla_{(a} \nabla_{b)}+\overline{\mathrm{P}}_{a b}\right) \overline{\mathbb{D}}_{A_{1}}^{B_{1}} \ldots \overline{\mathbb{D}}_{A_{\ell}}^{B_{\ell}}$, where $\nabla$ on the right hand side denotes the coupled affine-tractor connection.

5.2. Killing tensors in projective geometry. Let $\ell \in \mathbb{N}$. We shall focus on the PDE

$$
\nabla_{\left(a_{0}\right.} k_{\left.a_{1} \ldots a_{\ell}\right)}=0, \quad k_{a_{1} \ldots a_{\ell}} \in \mathcal{E}_{\left(a_{1} \ldots a_{\ell}\right)}(2 \ell),
$$

which is projectively invariant 9 .

Putting $\overline{\mathcal{E}}_{A}=\square$, we have $\overline{\mathcal{E}}_{[A B]}=\square$ and we set

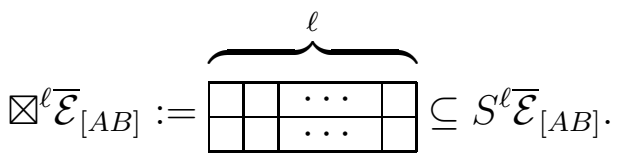

There exists a linear map $\bar{\Pi}^{(\ell)}: \mathcal{E}_{a_{1} \ldots a_{\ell}}(2 \ell) \rightarrow \nabla^{\ell} \overline{\mathcal{E}}_{[A B]}$, characterized by curved Casimir operators (see [6]), which takes the form

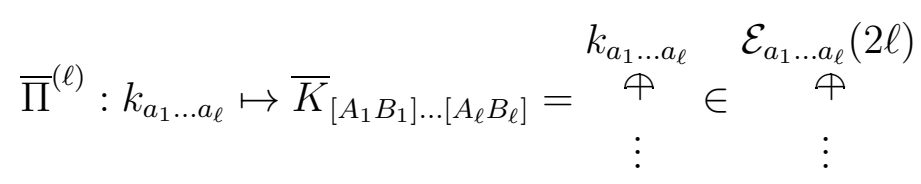

and such that $k_{a_{1} \ldots a_{\ell}}$ is a solution of (50) if and only if $\bar{K}_{\left[A_{1} B_{1}\right] \ldots\left[A_{\ell} B_{\ell}\right]}$ is $\bar{\nabla}$-parallel. Note the unspecified terms (indicated by vertical dots) of $\bar{K}_{\left[A_{1} B_{1}\right] \ldots\left[A_{\ell} B_{\ell}\right]}$ are differential in $k_{a_{1} \ldots a_{\ell}}$, i.e., the map $\bar{\Pi}^{(\ell)}$ is given by a differential operator. In fact, this is an example of a splitting operator (see e.g. [6] for details). It yields an analog of Theorem 4.9]

Proposition 5.2. [2] Let $(M,[\nabla])$ be a projectively flat manifold. The map $\bar{\Pi}^{(\ell)}$ induces a bijective correspondence

$$
\left\{\text { solutions } k_{a_{1} \ldots a_{\ell}} \text { of }(\underline{50})\right\} \stackrel{1-1}{\longleftrightarrow}\left\{\bar{\nabla}-\text { parallel sections of } \nabla^{\ell} \overline{\mathcal{E}}_{[A B]}\right\} \text {. }
$$


If the Levi-Civita connection of a metric $g$ pertains to the projective class $[\nabla]$, the latter proposition gives a description of Killing tensors for the metric $g$ via the map

$$
V^{\left(a_{1} \ldots a_{\ell}\right)} \mapsto g_{a_{1} b_{1}} \cdots g_{a_{1} b_{1}} V^{\left(a_{1} \ldots a_{\ell}\right)} \in \mathcal{E}_{\left(b_{1} \ldots b_{\ell}\right)}(2 \ell) .
$$

Indeed, this map gives a bijection between Killing $\ell$-tensors and solutions of the Equation (50).

5.3. Construction of symmetries. Now assume there is a LeviCivita connection $\nabla$ in the projective class $[\nabla]$, such that the associated metric $g_{a b}$ has constant curvature, i.e., $R_{a b c d}=\frac{4}{n} \mathrm{~J} g_{c[a} g_{b] d}$ with J parallel. Then a short computation based on (43) and (5) shows that $\bar{\nabla}$ on $\overline{\mathcal{E}}^{[A B]}$ (resp. $\overline{\mathcal{E}}_{[A B]}$ ) agrees with the Riemannian tractor connection $\nabla$ on $\mathcal{E}^{\mathbf{A}}$ (resp. $\mathcal{E}_{\mathbf{A}}$ ). Moreover the tractor section

$$
h^{A B}=\left(\begin{array}{c}
g^{a b} \\
0 \\
\frac{2}{n} \mathrm{~J}
\end{array}\right) \in \overline{\mathcal{E}}^{(A B)}=\begin{array}{cc}
\mathcal{E}^{(a b)}(-2) & \mathcal{E}^{(a b)} \\
\uparrow & \mathcal{E}^{a}(-2) \\
\uparrow & \stackrel{\mathcal{E}^{a}}{\oplus} \\
\mathcal{E}(-2) & \stackrel{\oplus}{\mathcal{E}}
\end{array}
$$

is parallel, cf. [11]. The isomorphism $\cong$ corresponds to the choice of connection $\nabla \in[\nabla]$, and in particular trivializes density bundles. Here $g^{a b}$ is the inverse of $g_{a b}$ and $\mathrm{J}=g^{a b} \mathrm{P}_{a b}=\frac{1}{2} g^{a b} \overline{\mathrm{P}}_{a b}$. Summarizing, we shall consider the Riemannian manifold $(M, g)$ as the corresponding locally flat projective manifold $(M,[\nabla])$ with the distinguished parallel section $h^{A B}$. This is an example of holonomy reduction of Cartan connections, [3], for the projective Cartan connection associated to $(M,[\nabla])$.

For $\mathrm{J} \neq 0$, note $h^{A B}$ is non-degenerate, hence a tractor metric. A direct computation gives the following display and lemma:

$$
h^{P[A} \overline{\mathbb{D}}_{P}^{B]} g^{a b}=h^{P[A} \overline{\mathbb{D}}_{P}^{B]} g_{a b}=0 .
$$

Lemma 5.3. The explicit formula for the differential operator

$$
h^{P[A} \overline{\mathbb{D}}_{P}{ }^{B]}: \mathcal{E}(w) \rightarrow \mathcal{E}^{[A B]}(w),
$$

written in terms of the Levi-Civita connection $\nabla$, does not depend on $w \in \mathbb{R}$.

We are ready now to construct the commuting symmetries of the Laplace operator. The metric $g$ allows to identify a tensor $V^{a_{1} \ldots a_{\ell}} \in$ $\mathcal{E}^{\left(a_{1} \ldots a_{\ell}\right)}$ with an element in $\mathcal{E}_{\left(a_{1} \ldots a_{\ell}\right)}(2 \ell)$, see $(53)$, and we denote by $\bar{V} \in \nabla^{\ell} \overline{\mathcal{E}}_{[A B]}$ the corresponding tractor, obtained via the map $\bar{\Pi}^{\ell}$ (see (51)). We consider the operators

$$
\overline{\mathcal{D}}^{V}:=h^{A_{1} C_{1}} \ldots h^{A_{\ell} C_{\ell}} \bar{V}_{A_{1} B_{1} \ldots A_{\ell} B_{\ell}} \overline{\mathbb{D}}_{C_{1}}^{B_{1}} \ldots \overline{\mathbb{D}}_{C_{\ell}}^{B_{\ell}}
$$

acting on any tensor-tractor bundle $U$. 
Lemma 5.4. The principal symbol of the differential operator $\overline{\mathcal{D}}^{V}$ is the symmetric $\ell$-tensor $V$.

Proof. The proof is analogous to the proof of Theorem 4.11. Writing tractor sections in the vertical notation (see (44)), we can refer to their "top" or "bottom" parts. The "top" part of $\bar{V}_{A_{1} B_{1} \ldots A_{\ell} B_{\ell}}$ is $g_{a_{1} b_{1}} \cdots g_{a_{1} b_{1}} V^{\left(a_{1} \ldots a_{\ell}\right)}$, cf. (51) and (53). On the other hand, an elementary computation (using (47) and (54)) shows that the "bottom" part (and the leading term) of $h^{C[A} \overline{\mathbb{D}}_{C}{ }^{B]} f$ is equal to $g^{a b} \nabla_{b} f$ for any section $f$ of a tensor bundle. Therefore, the "bottom" part of the com-

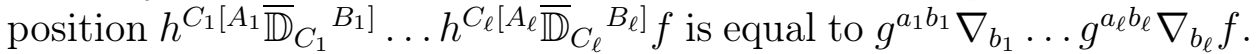
This completes the proof of the Lemma.

Theorem 5.5. Let $(M, g)$ be a pseudo-Riemannian manifold of constant curvature, with Levi-Civita connection $\nabla$, and $(M,[\nabla])$ the corresponding locally flat projective manifold. Then, if $k$ is a Killing $\ell-$ tensor, the operator $\overline{\mathcal{D}}^{k}: \mathcal{E} \rightarrow \mathcal{E}$, defined by (156), is a commuting symmetry of the Laplace operator $\Delta=g^{a b} \nabla_{a} \nabla_{b}$.

Proof. Let $\bar{K} \in \nabla^{\ell} \overline{\mathcal{E}}_{[A B]}$ be the parallel tractor associated to $k$ via the composition of the maps (53) and (52). Since the tractor metric $h$ is also parallel with respect to the projective tractor connection $\bar{\nabla}$, it follows from (49) and (55) that

$$
\overline{\mathcal{D}}^{k}\left(g^{a b}\left(\nabla_{(a} \nabla_{b)}+\overline{\mathrm{P}}_{a b}\right)\right)=\left(g^{a b}\left(\nabla_{(a} \nabla_{b)}+\overline{\mathrm{P}}_{a b}\right)\right) \overline{\mathcal{D}}^{k}: \mathcal{E}(+1) \rightarrow \mathcal{E}(-1),
$$

where we consider $g^{a b} \in \mathcal{E}^{(a b)}(-2)$. The operator $\overline{\mathcal{D}}^{k}: \mathcal{E}(w) \rightarrow \mathcal{E}(w)$, expressed in terms of $\nabla$, does not depend on $w \in \mathbb{R}$ by Lemma 5.3 . Observing that $g^{a b} \overline{\mathrm{P}}_{a b}$ is parallel for $\nabla$, the theorem follows.

Remark 5.6. Projectively invariant overdetermined operators, as the operator defined in (48), are discussed in [9]. They allow for analogous construction of symmetries for other Riemannian linear differential operators $F: \Gamma(U) \rightarrow \Gamma(U)$.

Acknowledgements: JPM is supported by the Belgian Interuniversity Attraction Pole (IAP) within the framework "Dynamics, Geometry and Statistical Physics" (DYGEST). PS and JS gratefully acknowledge the support of the grant agency of the Czech Republic under the grant P201/12/G028.

\section{REFERENCES}

[1] T. N. Bailey, M. G. Eastwood, A. R. Gover, Thomas's structure bundle for conformal, projective and related structures, Rocky Mountain J. Math. 24 (1994), no. 4, 1191-1217.

[2] T. Branson, A. Čap, M. G. Eastwood, A. R. Gover, Prolongations of geometric overdetermined systems. Internat. J. Math. 17 (2006), no. 6, 641-664. 
[3] A. Čap, A. R. Gover, M. Hammerl, Holonomy reductions of Cartan geometries and curved orbit decompositions, electronically available as Preprint ESI 2308.

[4] A. Čap, A. R. Gover, M. Hammerl, Projective BGG equations, algebraic sets, and compactifications of Einstein geometries, J. London Math. Soc. 86 no. 2 (2012) 433-454.

[5] A. Čap, J. Slovák, Parabolic geometries I: Background and general theory, Mathematical Surveys and Monographs 154. Providence, RI: American Mathematical Society (AMS), 628 pp.

[6] A. Čap, V. Souček, Curved Casimir operators and the BGG machinery, SIGMA Symmetry Integrability Geom. Methods Appl. 3 (2007), Paper 111, $17 \mathrm{pp}$.

[7] B. Carter. Killing tensor quantum numbers and conserved currents in curved space. Phys. Rev. D (3), 16(12):3395-3414, 1977.

[8] M. G. Eastwood, Higher Symmetries of the Laplacian, Ann. Math., 161 (2005), 1645-1665.

[9] M. G. Eastwood, A. R. Gover, The BGG complex on projective space. SIGMA Symmetry Integrability Geom. Methods Appl. 7 (2011), Paper 060, 18 pp.

[10] G. B. Folland, Harmonic analysis in phase space, volume 122 of Annals of Mathematics Studies. Princeton University Press, Princeton, NJ, 1989.

[11] A. R. Gover, H. Macbeth, Detecting Einstein geodesics: Einstein metrics in projective and conformal geometry, Archive http://arxiv.org/abs/1212.6286.

[12] A. R. Gover, J. Šilhan, Higher symmetries of the conformal powers of the Laplacian on conformally flat manifolds, J. Math. Phys. 53 (2012), no. 3, 032301, 26 pp.

[13] B. Kostant, Verma modules and the existence of quasi-invariant differential operators, in Non-commutative Harmonic Analysis, Lect. Notes Math. 466, 101-128, Springer Verlag, New York, 1975.

[14] B. Kostant, Holonomy and the Lie algebra of infinitesimal motions of a Riemannian manifold, Trans. Amer. Math. Soc. 80 (1955), 528-542.

[15] R. G. McLenaghan, R. Milson, R. G. Smirnov, Killing tensors as irreducible representations of the general linear group. C. R. Math. Acad. Sci. Paris 339 (2004), no. 9, 621-624.

[16] J.-Ph. Michel. Higher symmetries of Laplacian via quantization, to appear in Ann. Inst. Fourier, Archive http://arxiv.org/abs/1107.5840.

[17] M. Takeuchi, Killing tensor fields on spaces of constant curvature. Tsukuba J. Math. 7 (1983), no. 2, 233-255.

[18] J. A. Wolf, Spaces of constant curvature, McGraw-Hill in New York, 1967.

JPM: University of Liège, Grande Traverse, 12, Sart-Tilman, B-4000

LiĖGe, BELGiUM

E-mail address: jean-philippe.michel@ulg.ac.be

PS: Mathematical Institute, Charles University, Sokolovská 83, Prague, Czech Republic

E-mail address: somberg@karlin.mff.cuni.cz

JS: Institute of Mathematics and Statistics, Masaryk University, Building 08, KotlářsKá 2, 611 37, Brno, Czech Republic

E-mail address: silhan@math.muni.cz 